First published in:

\title{
Influence of trees on the dispersion of pollutants in an urban street canyon-Experimental investigation of the flow and concentration field
}

\author{
Christof Gromke*, Bodo Ruck \\ Laboratory of Building and Environmental Aerodynamics, Institute for Hydromechanics, University of Karlsruhe, Kaiserstr. \\ 12, 76128 Karlsruhe, Germany
}

Received 11 August 2006; received in revised form 14 November 2006; accepted 21 December 2006

\begin{abstract}
Flow field and concentration measurements have been performed in an idealized model of an urban street canyon with one row of trees arranged along the center axis. The model was set up in an atmospheric boundary layer wind tunnel and the approach flow was directed perpendicular to the street axis. A line source embedded in the bottom of the street was used to release tracer gas for the simulation of traffic exhaust emissions. Trees with spherical crowns were modeled and positioned inside the street canyon, varying crown diameter, crown permeability, trunk height and tree spacing. Trafficinduced turbulence was simulated by rotating belts with thin plates. Concentrations were measured at the facades of the street canyon. For small tree crowns, only little changes in concentration were measured, however, increasing crown diameters led to increasing concentrations at the leeward street canyon wall associated with a reduction of local concentrations at the windward wall. For some cases, a variation of trunk height led to a modification of the concentration pattern on the walls. Increasing the tree spacing resulted in a noticeable concentration decrease. When compared to the situation with standing (but emitting) traffic, the traffic-induced turbulence by two-way car movements always contributed to a more homogenous concentration field inside the street canyon yielding to reduced mean concentration levels.
\end{abstract}

(C) 2007 Elsevier Ltd. All rights reserved.

Keywords: Street canyon; Pollutant dispersion; Trees; Canyon vortex; Traffic-induced turbulence

\section{Introduction}

The dilution and removal of car exhaust emissions released in city centers is of great importance for the health and quality of life of urbanities. In general, air exchange in towns is provided by natural ventilation. Beside the wind field flowing

\footnotetext{
*Corresponding author. Tel.: + 497216087307 ; fax: +497216083191 .

E-mail address: gromke@ifh.uka.de (C. Gromke).
}

over the canyon, a sufficient natural ventilation depends on the street canyon shape, i.e. the aspect ratio of canyon width to canyon depth. There is quite a number of experimental and numerical studies dedicated to street canyons and the flow and transport mechanisms are well understood (Kastner-Klein and Plate, 1999; Chang and Meroney, 2003; So et al., 2005; Eliasson et al., 2006; Gerdes and Olivari, 1999; Baik and Kim, 1999). Recently, some reviews on wind tunnel studies of dispersion phenomena in street canyons 
were published by Vardoulakis et al. (2003) and Ahmad et al. (2005). However, the influence of obstacles like trees on dispersion phenomena inside an urban street canyon is disregarded in common investigations. The impact of obstacles on the flow field and pollutant dispersion inside a street canyon or cavity was addressed only in few studies. Gayev and Savory (1999) experimentally investigated the influence of regularly arranged vertical cylinders in a cavity under perpendicular oncoming flow conditions. They found the mean flow velocity in the cavity to be reduced and the turbulence intensity to be increased by $50-200 \%$ when compared to the reference case without cylinders. Furthermore, they observed a somewhat different form of the rotating vortex system in the canyon. In a numerical study Ries and Eichhorn (2001) investigated the influence of trees planted in two rows parallel to the street axis. Using a $k-\varepsilon$ turbulence model and taking the permeability of trees into account, they found at the leeward wall local increases of pollution level accompanied by reduced flow velocities. However, they applied a two-dimensional model and their results do not account for three-dimensional effects, e.g. induced by corner eddies.

The aim of this study is to clarify the impact of trees on the natural ventilation and pollutant dispersion within a street canyon. For this reason, wind tunnel experiments at an idealized urban street canyon with model trees arranged inside have been performed. Tree characteristics like crown diameter, crown permeability, crown height and tree spacing have been varied and corresponding flow and concentration fields were measured. In order to acquire the influence of vehicle motion on pollutant dispersion, additional measurements with simulation of traffic-induced turbulence have been conducted. The practical intention of all these investigations is to provide city planers with guidelines on how to plant trees in urban street canyons in regard to air quality management.

\section{Model setup and measurement instrumentation}

The influence of trees on flow and dispersion phenomena investigated in a three-dimensional wind tunnel model of an urban street canyon of scale 1:150 is discussed (Fig. 1). In reality, the canyon has a width and depth of $18 \mathrm{~m}$ and a length of $180 \mathrm{~m}$. Trees with spherical crowns of varying diameter ( 9 and $15 \mathrm{~m}$ ) consisting of impermeable Styrofoam or permeable foam 10 ppi (10 pores per

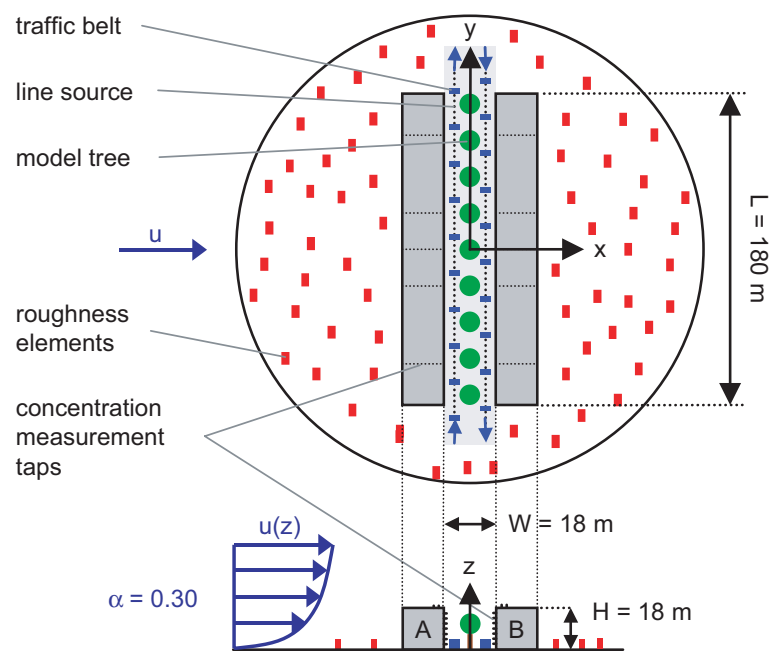

Fig. 1. Wind tunnel model of the urban street canyon (scale $1: 150)$.

inch) were modeled and placed along the canyon center axis inside the urban street canyon. Furthermore, the height of the branch free trunk was varied $(4.5$ and $9 \mathrm{~m})$ as well as the tree spacing (15 and $20 \mathrm{~m})$. Investigations have been carried out for standing traffic and two-way traffic with vehicle velocities of $40 \mathrm{~km} \mathrm{~h}^{-1}$.

The boundary layer wind tunnel used for the experiments has a cross section of $2 \mathrm{~m} \times 1 \mathrm{~m}$ (width $\times$ height) and an adjustable ceiling to compensate for pressure losses. After passing vortex generators and a $5 \mathrm{~m}$ long fetch covered with roughness elements, a stable wind profile has developed which is characterized by the exponent $\alpha=0.30$ according to the power law of the velocity profile. Flow and concentration measurements have been performed with a flow velocity of $7 \mathrm{~m} \mathrm{~s}^{-1}$ at the boundary layer height of the undisturbed inlet profile, corresponding to a velocity of $4.6 \mathrm{~m} \mathrm{~s}^{-1}$ at roof top level and ensuring a Reynolds number independent flow field, see also Gromke and Ruck (2005). The aspect ratio $H / W$ (building height $H$ to street width $W$ ) of the urban street canyon is given by 1 and the $L / W$-ratio (street length $L$ to street width $W$ ) equals 10 . Thus, according to Oke (1988) and Hunter et al. (1990/1991) the prevailing flow field is that of a skimming flow regime.

Sulfur hexafluoride $\left(\mathrm{SF}_{6}\right)$ was used as tracer gas, which was emitted homogenously by a line source mounted at the bottom of the model (Meroney et al., 1996). The street canyon model was equipped with 90 concentration measurement taps. For analyzing the mean tracer gas concentration, an 
electron capture detector (ECD) (model: Meltron LH 108) was used. Investigations concerning the accuracy of concentration measurements were done by comparison of repeated measurements and yield for wall $\mathrm{A}$, a mean deviation of $m_{\mathrm{A}}=4.1 \%$ and a standard deviation of $\sigma_{\mathrm{A}}=2.1 \%$. For the lower polluted wall $\mathrm{B}$ the corresponding values were $m_{\mathrm{B}}=6.4 \%$ and $\sigma_{\mathrm{B}}=5.1 \%$.

Additionally, the flow field has been analyzed by two-component laser Doppler velocimetry (LDV). Since LDV-measurements inside the street canyon were mostly impossible due to obstruction by tree crowns, flow velocities have been acquired only at the boundaries above and sidewise of the canyon. These measurements have been used to calculate the air volume fluxes entering and leaving the canyon and permit statements on essential changes in the flow field in the presence of different tree configurations. Furthermore, these velocity values provide a database for the calibration and validations of numerical computations.

Traffic-induced turbulence was simulated by small plates mounted on rotating belts (see Fig. 20) and modeled according to a similarity criteria, which states that the ratio of turbulence generated by atmospheric wind overflowing the surrounding buildings to the one generated by traffic has to be equal in large and small scale (Plate, 1982). Denoting this ratio by $T_{P}$, the turbulence generated by interaction of atmospheric flow and buildings by $P_{W}$ and the traffic-induced turbulence by $P_{T}, T_{P}$ can be expressed as

$T_{P}=P_{T} / P_{W} \propto \frac{\rho c_{\mathrm{D}} u_{T}^{3} n_{T} F_{T}}{W H} / \frac{\rho c_{\mathrm{f}} u_{\delta}^{3}}{H}$,

with $\rho$ density of air, $c_{\mathrm{D}}$ drag coefficient of cars, $u_{T}$ traffic velocity, $F_{T}$ frontal area of cars, $n_{T}$ traffic density (cars/length), $c_{\mathrm{f}}$ friction coefficient of city canopy, $u_{\delta}$ flow velocity at boundary layer height, $W$ street width and $H$ height of buildings.

As mentioned before, in the wind tunnel experiments cars were modeled by plane plates mounted on rotating belts. These plates have drag coefficients of $c_{\mathrm{D}}=1.2$ and frontal areas of $F_{T}=50 \mathrm{~mm}^{2}$, each. For the following measurements either a two-way traffic motion with $40 \mathrm{~km} \mathrm{~h}^{-1}$ and a traffic density of $n_{T}=6.67$ cars $/ 100 \mathrm{~m}$ in presence of a wind velocity in boundary layer height of $u_{\delta}=7 \mathrm{~m} \mathrm{~s}^{-1}$, denoted by TWT40, or the situation of standing but exhaust emitting traffic, denoted by ST00, were simulated. In both the traffic situations, the line source strengths were equal.
Usually, results of concentration measurements are presented in form of non-dimensional concentrations given by

$c^{+}=\frac{c_{\text {meas }} u_{\text {ref }} L_{\mathrm{ref}}}{Q_{\mathrm{T}} / l}$,

with $c_{\text {meas }}$ measured concentration, $u_{\text {ref }}$ reference velocity characterizing the atmospheric flow, $L_{\text {ref }}$ reference length characterizing the dimensions of buildings and $Q_{\mathrm{T}} / l$ as tracer gas emission rate of the line source. Since the flow just above the roof top level is driving the canyon vortex and, thus, providing air exchange in the middle part of the canyon, the velocity at roof top height of the undisturbed approach flow was chosen as $u_{\text {ref }}$. Further, the building height $H$ was chosen as $L_{\text {ref }}$.

In the following figures, the concentrations at building walls $\mathrm{A}$ and $\mathrm{B}$ predominantly display the relative change in percentage when compared to a reference configuration. This allows to easily visualize changes in the concentration distribution due to a certain variation, e.g. traffic or tree planting, and simplifies the understanding of the impact. The concentration distributions were given in the range of $-3.75<y / H<+3.75$, which corresponds to the positions of the outermost concentration measurement taps.

\section{Measurement results and interpretation}

In Fig. 2, the situation of a typical urban street canyon subjected to perpendicular approaching flow is shown. Two dominating flow phenomena can be seen, a canyon vortex $(\mathrm{CV})$ in the middle part of the street canyon and corner eddies (CE) at the ends of the street canyon. Whereas the horizontally rotating canyon vortex, driven by shear forces of the

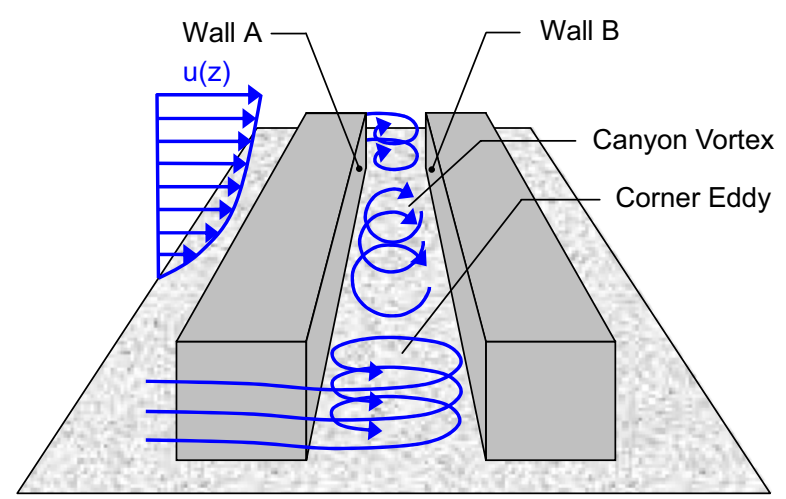

Fig. 2. Flow field in and around an urban street canyon. 
skimming flow above the roof top level is the only source of air exchange in the canyon center part, towards the street ends, additional air exchange is provided by vertically rotating corner eddies. The superposition of both at the street ends yields to a more three-dimensional flow field and, of course, to a better ventilation. Hunter et al. (1990/1991) found by numerical studies using a $k-\varepsilon$ turbulence closure that for an urban street canyon exhibiting an aspect ratio $H / W=1$, the canyon middle region is subjected to discrete canyon vortices only when $L / H \geqslant 7$. Thus, in the present case with $L / H=10$, a clear distinct zone with canyon vortices can be identified.

\subsection{Reference case without trees-influence of traffic motion}

\subsubsection{Reference case without trees and standing traffic (STO0)}

In Fig. 3, contour maps for dimensionless concentrations at wall $\mathrm{A}$ and wall $\mathrm{B}$ of the reference case, i.e. without trees and standing but exhaust emitting traffic (ST00) are shown. These results are in qualitatively good agreement with the wind tunnel measurements of Kastner-Klein et al. (2001). The average concentration values at wall A are approximately 2.5 times higher than at wall B, which can be easily explained by regarding the flow regime inside and above the street canyon. Driven by the skimming flow over the roof top, a canyon vortex rotating in clockwise direction is present between wall A and B. Clean air of the skimming flow gets partially mixed into the street canyon after passing the street centerline and diverts towards the ground in front of wall B. On the reverse flow from wall $\mathrm{B}$ towards wall $\mathrm{A}$ the clean air accumulates exhaust emissions released by traffic. Thus, the ascending air in front of wall A exhibits higher concentrations, especially in the lower part at the pedestrian level, before it gets mixed up with fresh air of the incident flow at roof top level. Towards the street corners, a decrease of concentration level for both walls is observable, which is due to an additional ventilation induced by the corner eddies.

The corresponding mean vertical component $w$ of the flow field above the street canyon in a vertical plane along the wind direction at three different positions of $y / H$ is displayed in the contour maps of Fig. 4. Regarding the vertical velocity component $w$ shows zones of positive and negative flow velocities, i.e. air is coming out or entering the canyon, respectively. In accordance with the before mentioned prevailing flow regime in the middle part of the street canyon, a positive vertical velocity component $w$ in the first half $(-0.5 \leqslant x / H \leqslant 0)$ and a negative velocity component $w$ in the second half $(0 \leqslant x / H \leqslant 0.5)$ is identifiable for the normalized cross section positions $y / H=0$ and \pm 2.5 . At position $y / H= \pm 5$ (corner edge) the flow above the roof top shows consistently a negative value for $w$, due to superposition by corner eddies the flow field becomes more three dimensional and the canyon vortex is no longer the solely acting flow phenomenon.

A more comprehensive impression of the flow field above the street canyon can be obtained by means of vector maps in Fig. 5, showing the same cross sections as in Fig. 4. In the vector plot for position $y / H=0$, the upper part of the clockwise
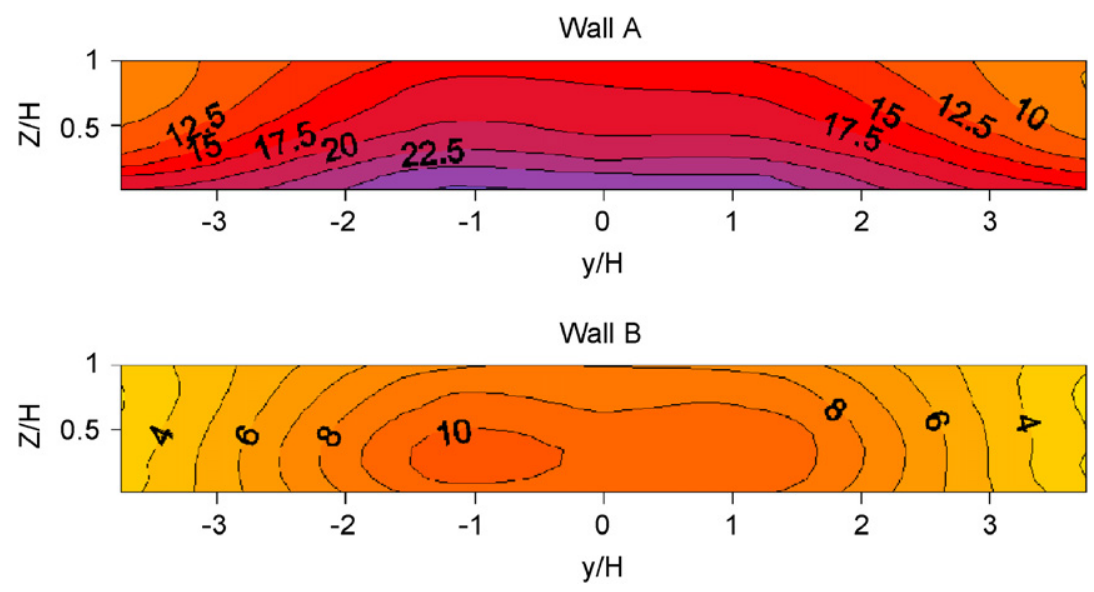

Fig. 3. Dimensionless wall concentrations for reference case without trees with standing traffic (ST00). 

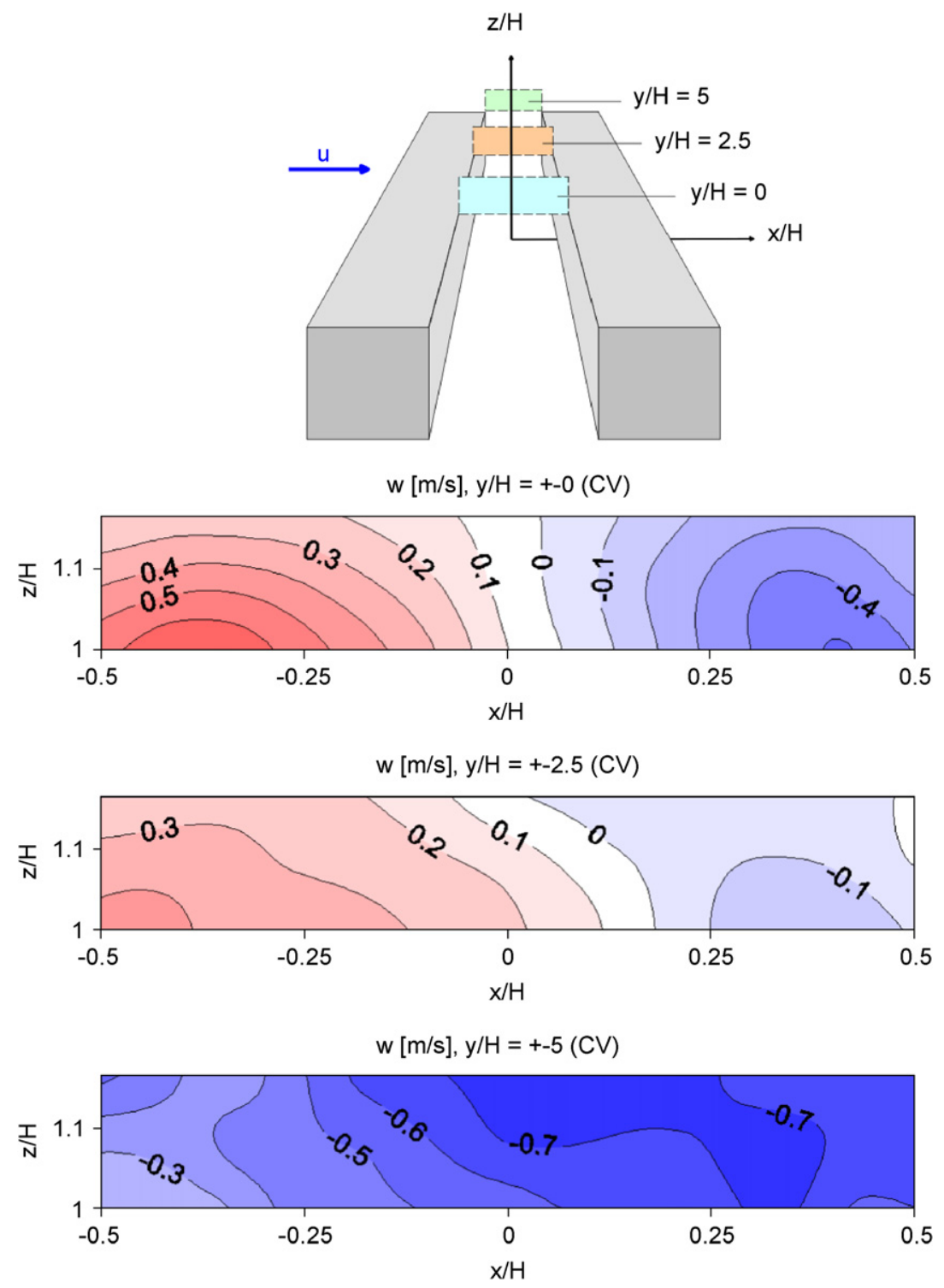

Fig. 4. Mean vertical velocity $w$ in vertical planes over the street canyon for reference case with standing traffic (ST00).

rotating canyon vortex and a gradual transition into the atmospheric shear flow above the roof top can be inferred. At positions $y / H= \pm 5$, the flow over the canyon is dominated by the sidewise entering corner eddies.

Finally, in Fig. 6, the mean horizontal velocity component $v$ in the end cross sections of the street canyon at $y / H= \pm 5$ are presented, with positive values indicating flow entering and negative values indicating flow coming out of the canyon. The corner eddies, which separate at windward building A, enter the street canyon showing a somewhat eccentric center shifted to building B. Summing up the in- and outflow rates through the end cross sections, a net inflow is remaining. This sidewise inflow mass flux must flow off through the horizontal roof plane of the street canyon. Regarding the contour map of the $w$ component at position $y / H= \pm 2.5$ in Fig. 4 obviously reveals a net outflow rate.

\subsubsection{Reference case without trees and two-way traffic (TWT40)}

The measurement results of the same experimental setup, but with additional two-way traffic movement at $40 \mathrm{~km} \mathrm{~h}^{-1}$ are shown in Fig. 7. 

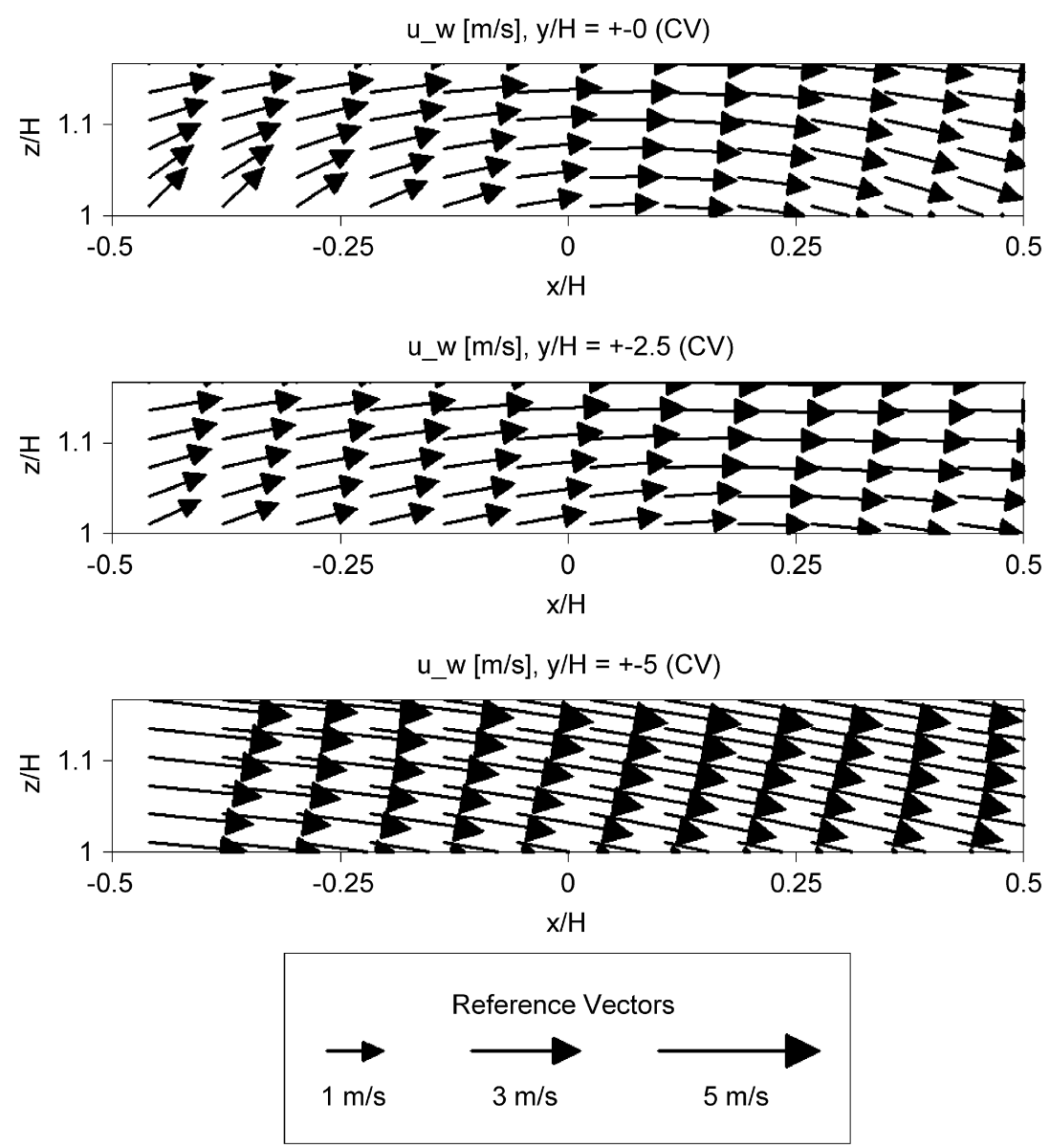

Fig. 5. Vector maps of flow fields in vertical planes above the street canyon for reference case with standing traffic (ST00).

When comparing Fig. 7 with Fig. 3, lower peak values and a more homogeneous concentration distribution for the traffic case can be seen easily. These impacts of traffic-induced turbulence caused by two-way traffic have been observed by KastnerKlein et al. (2001), too. In order to facilitate the understanding of the impact of traffic-induced turbulence, the relative percentage change in concentration in comparison to the reference case with standing traffic (ST00), see Fig. 3, is shown in Fig. 8. At wall $\mathrm{A}$, a small decrease in the middle part and an increase of tracer gas concentration towards the corners is detected. This can be explained by the traffic action which provides an enhanced mixing of vehicle exhausts in the street canyon by transporting higher polluted air from the canyon middle part to lower polluted areas at the street ends and vice versa. The same phenomena is present at wall $\mathrm{B}$, however, due to intrinsic lower concentration levels but equal traffic strength, the percentage of concentration decrease in the middle of this wall is more distinct.

With regard to the $w$ component of the flow field in the vertical planes above the street canyon, a comparison with the standing traffic case of Fig. 4 reveals that there is no significant change observable, i.e. the traffic-induced flow field at street level is not strong enough to influence the flow field above roof top.

In Fig. 9, the horizontal velocity component $v$ for two-way traffic (TWT40) in the canyon end cross sections at $y / H=-5$ is shown (analogue to Fig. 6). The values of Fig. 6 have not changed much. Even for lower $z / H$ positions no significant change of the velocity $v$ is visible, indicating that the influence of moving traffic on the inflow and outflow conditions at the canyon end cross sections is not very strong. 
Resuming the effect of two-way traffic motion in an open street canyon, it can be stated that there is only a small influence on the overall velocity field, whereas the influence on the concentration field is of considerable importance.
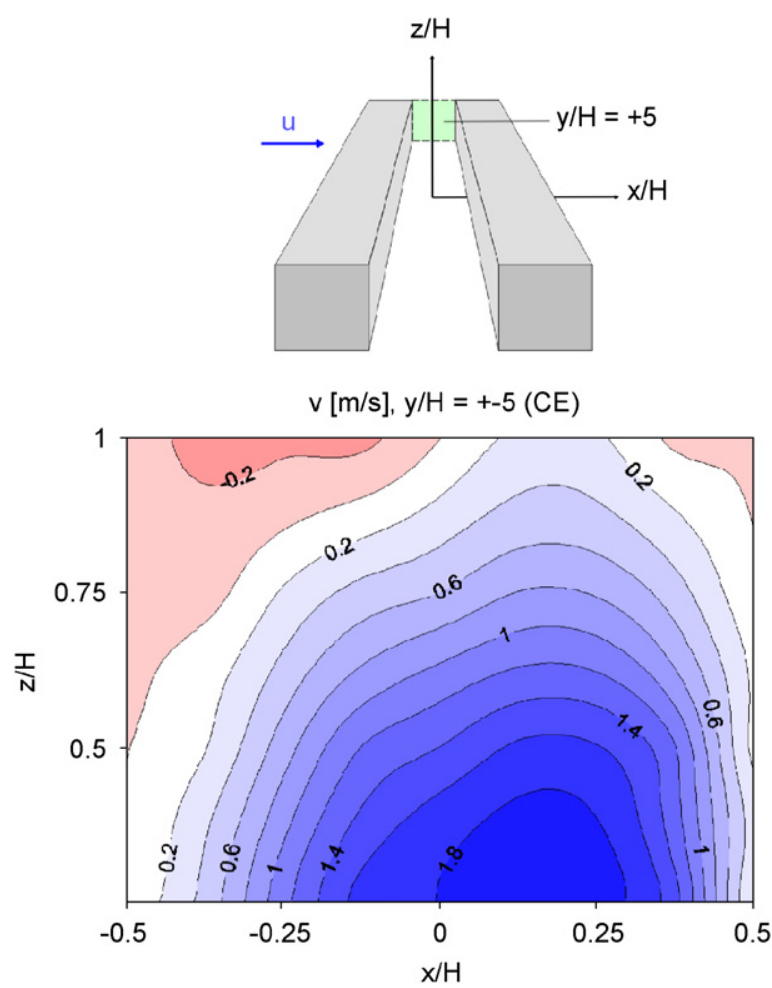

Fig. 6. Mean horizontal velocity $v$ at $y / H= \pm 5$ for reference case with standing traffic (ST00), positive values indicate flow into the canyon and negative values indicate flow out of the canyon.

\subsection{Influence of crown diameter}

3.2.1. Trees with spherical impermeable crowns of $9 \mathrm{~m}$ diameter and standing traffic (STOO)

With this series, impermeable spherical trees with crowns made out of Styrofoam and branch free trunk heights of $4.5 \mathrm{~m}$ were arranged in the middle axis of the street canyon between both traffic lanes at a distance of $15 \mathrm{~m}$ occupying $8.5 \%$ of the street canyon volume. Again, the relative percentage change in concentration related to the reference case of Fig. 3 is shown. The concentration level at the middle part of wall $\mathrm{A}$ is hardly affected by the tree planting as can be seen in Fig. 10. However, towards the canyon ends, a steady increase in concentration is found. For wall B a decrease in the center part and an increase in concentration at the corners are noticeable.

Regarding the velocity plot of Fig. 11 indicates that a rotation of fluid mass in the middle part of the canyon is still present. Since there is a gap of $6 \mathrm{~m}$ between the tree crowns and of $3 \mathrm{~m}$ between tree crowns and the walls, sufficient free space remains to form smaller but multiple canyon vortices. Consequently, the concentration level in the middle of the canyon at wall $\mathrm{A}$ is not strongly affected by the tree planting of the chosen kind.

The decrease in the middle part of wall B is not as easy to explain. However, having in mind that the concentrations found at wall $\mathrm{B}$ originate from traffic emissions which have been transported by the canyon vortex towards wall $\mathrm{B}$, a strong impact of the flow conditions in the roof top region on the dilution of the canyon vortex air is evident. Comparing the contour velocity plots of Fig. 11

Wall $A$

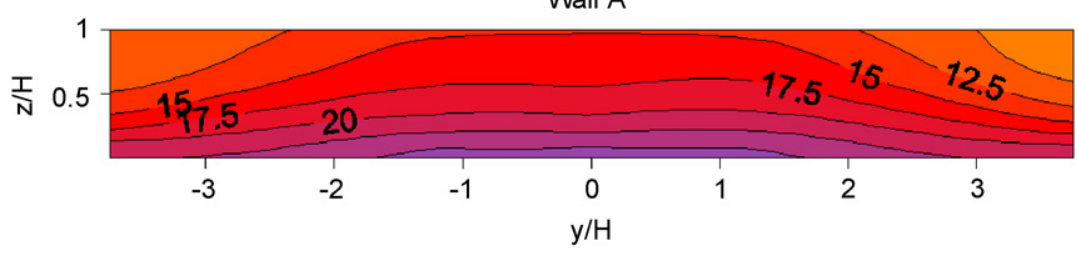

Wall B

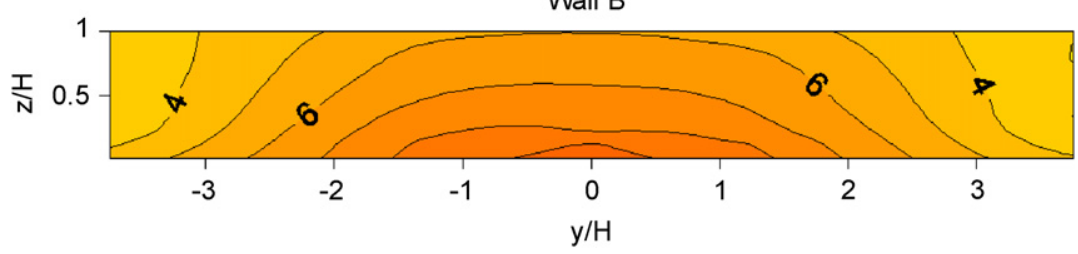

Fig. 7. Dimensionless wall concentrations for street canyon without trees and two-way traffic (TWT40). 

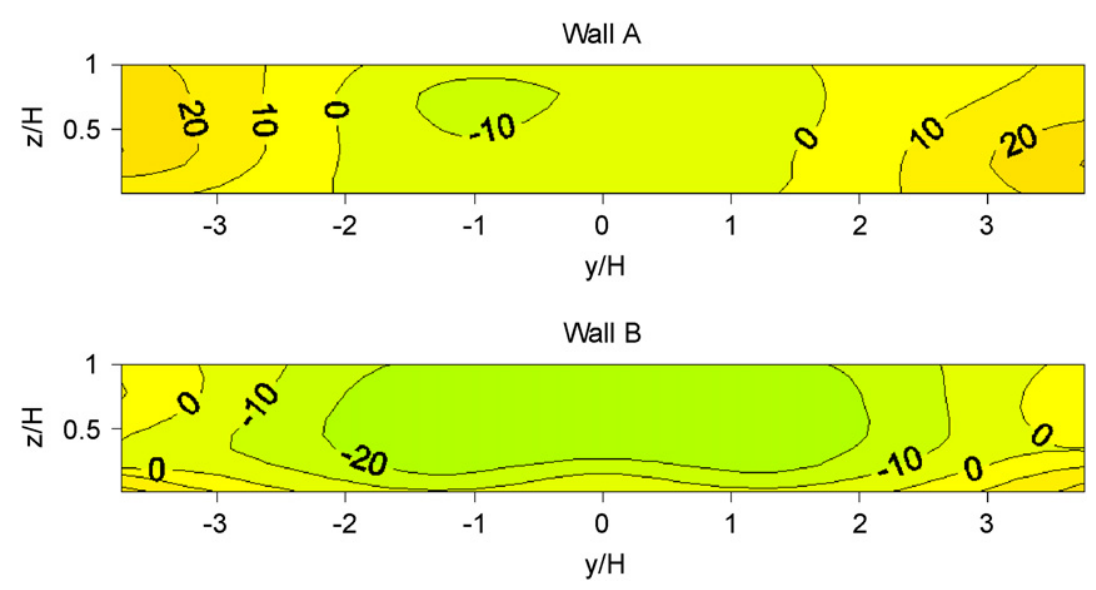

Fig. 8. Relative change in wall concentrations for street canyon without trees and two-way traffic (TWT40) when compared to case with standing traffic (ST00), see Fig. 3.

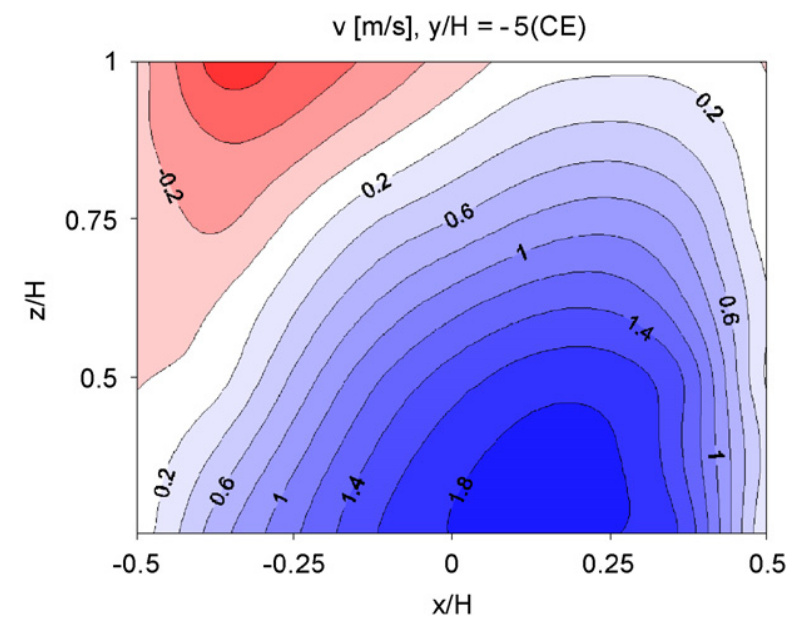

Fig. 9. Mean horizontal velocity $v$ at $y / H=-5$ for street canyon with two-way traffic (TWT40).

and of the reference case (Fig. 4) reveals somewhat higher vertical flow velocities $v$ in the downdraft region (top right) for the street canyon configuration with trees. That is, more fresh air from higher layers is entrained in the canyon vortex in front of wall $\mathrm{B}$, leading to lower concentrations.

The increase of concentration towards the canyon ends can be explained by the crowns of the outermost standing trees, which constitute obstacles or rather resistances and hinder the laterally flow induced by corner eddies entering into the street canyon. Comparing the contour map of the horizontal velocity component $v$ in the cross section at $y / H=-5$ of Fig. 12 with the one of the reference case (Fig. 6) shows smaller flow velocities associated with reduced inflow. Thus, a very effective pollutant dispersion and dilution mechanism is significantly weakened.

\subsubsection{Trees with spherical impermeable crowns of $9 \mathrm{~m}$ diameter and two-way traffic (TWT40)}

In the following, the same configuration as before was measured but with additional two-way traffic of $40 \mathrm{~km} \mathrm{~h}^{-1}$. The concentration maps for wall $\mathrm{A}$ and $\mathrm{B}$ are given in Fig. 13 and show the relative percentage change in concentration referred to the model configuration without trees, but two-way traffic at $40 \mathrm{~km} \mathrm{~h}^{-1}$, see Fig. 7 .

Again, the traffic-induced turbulence is leading to a more homogeneous distribution of exhaust emissions with smaller horizontal concentration gradients at both walls. There is only a small relative increase of wall A concentrations towards the canyon outer edges, i.e. the chosen configuration of tree planting does not exert a significant influence on the wall A concentrations. However, for wall B, a more pronounced increase in concentration is registered. This indicates that the tree crowns hinder the pollutants to be effectively removed, when compared to the no tree situation. Note that the concentration increases at the canyon ends are not symmetrically, but single-edged in direction of the traffic motion next to the wall.

Since no significant differences in the flow field above the roof top level due to traffic movement are detectable when compared to Fig. 11, the corresponding contour map showing the vertical velocity component $w$ is omitted.

From Fig. 14, where the distribution of the horizontal velocity component $v$ in the canyon 

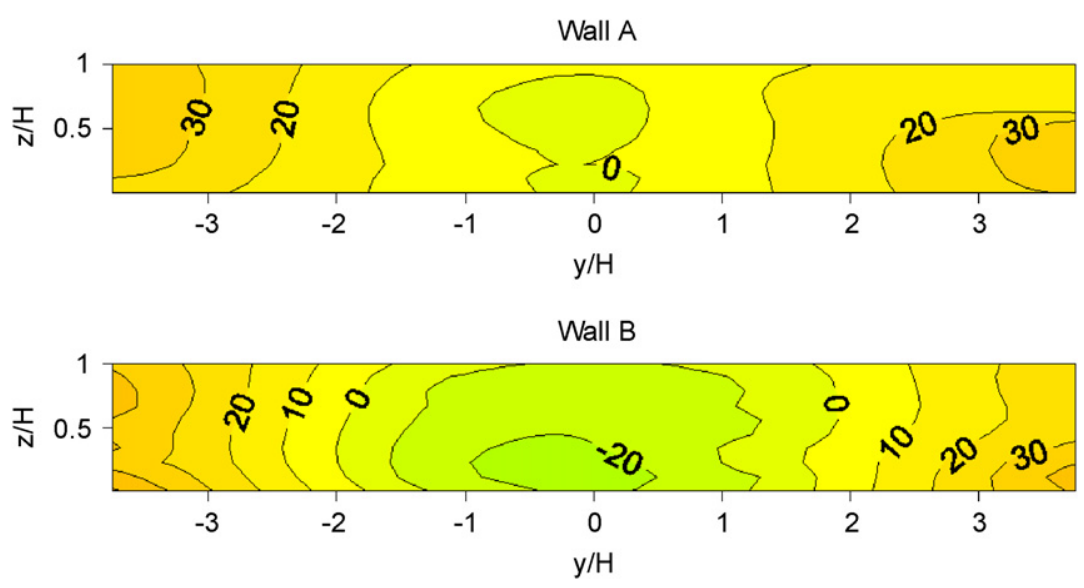

Fig. 10. Relative change in wall concentrations for street canyon with trees of $9 \mathrm{~m}$ crown diameter and standing traffic (ST00) when compared to no tree situation (Fig. 3).

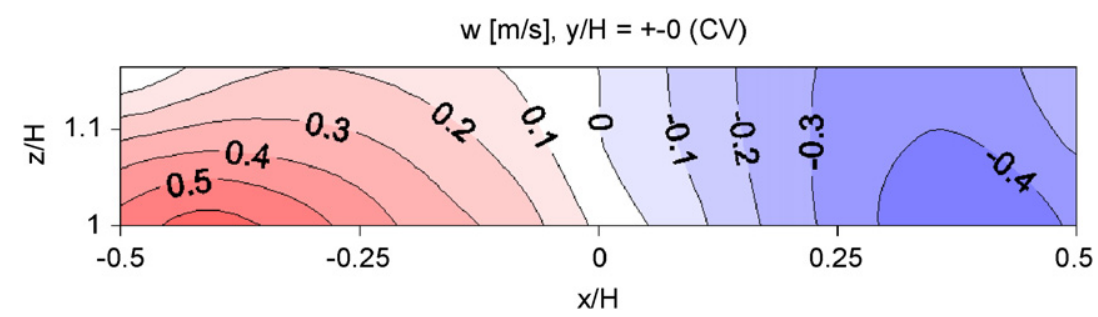

Fig. 11. Mean vertical velocity $w$ above street canyon with trees of crown diameter $9 \mathrm{~m}$ and standing traffic (ST00).

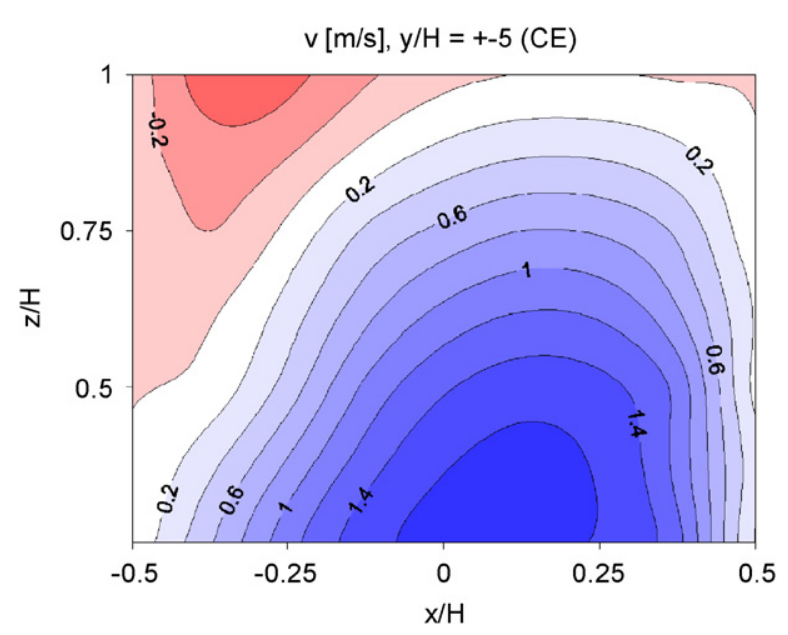

Fig. 12. Mean horizontal velocity $v$ at $y / H= \pm 5$ for street canyon with trees of crown diameter $9 \mathrm{~m}$ and standing traffic (ST00).

outermost cross sections at $y / H=-5$ is shown, it can be inferred that the tree planting in a canyon with two-way traffic alters significantly the in- and outflow conditions when compared to Fig. 9.
3.2.3. Trees with spherical impermeable crowns of $15 \mathrm{~m}$ diameter and standing traffic (STOO)

The tree spacing of $15 \mathrm{~m}$ remained unchanged for this series. The crown volume now occupies $39.4 \%$ of the street canyon volume. With this configuration, neighboring tree crowns are touching each other at their waists. The minimum branch free trunk height is $4.5 \mathrm{~m}$ and the treetops are overtopping the roof level by $1.5 \mathrm{~m}$. In Fig. 15 the relative change in concentration related to the reference case without trees (Fig. 3) is shown. At wall $\mathrm{A}$, a significant increase of tracer gas wall concentration in the middle section for $-2<y / H<2$ is observable, indicating that the canyon vortex strength has been reduced. Towards the edges, a strong increase of concentration is visible for both walls A and B because of the outermost tree crowns which are now significantly hindering the corner eddies by entering the street canyon. This shows again the important role of corner eddies in pollutant removal and dilution at the street ends. Wall B shows a concentration decline in the upper half of its middle part, which is found also in the case of trees with $9 \mathrm{~m}$ crown diameter, see Fig. 10 . Apparently, the tree planting changes the flow field 
Wall A

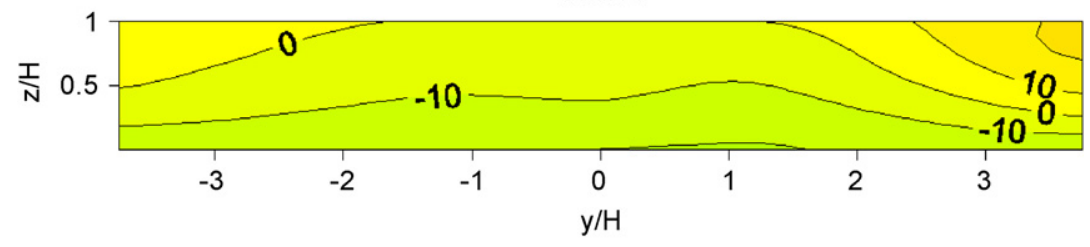

Wall B

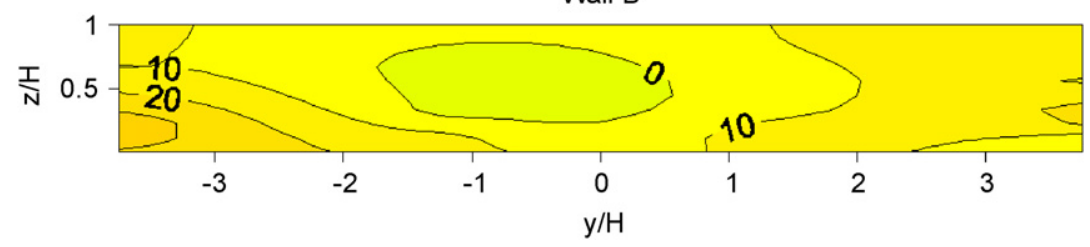

Fig. 13. Relative change in wall concentrations for street canyon with trees of crown diameter $9 \mathrm{~m}$ and two-way traffic (TWT40) when compared to no tree situation.

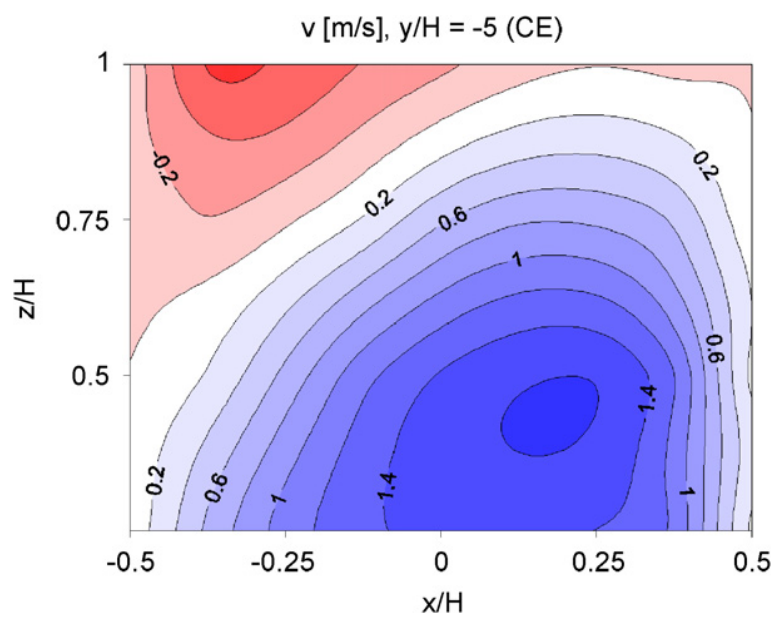

Fig. 14. Mean horizontal velocity $v$ at $y / H=-5$ for street canyon with trees of crown diameter $9 \mathrm{~m}$ and two-way traffic (TWT40).

in the roof top region and the entrainment conditions as discussed for the trees with $9 \mathrm{~m}$ crown diameter. As a consequence, smaller concentrations at the upper half of wall $\mathrm{B}$ when compared to the no tree situation are measured.

An indication for this assumption is also given in Fig. 16, where the vertical component $w$ of the flow field $1.5 \mathrm{~m}$ above the roof top level is depicted. A zone of air coming out of the street canyon to the left of the center axis and a zone of air entering the street canyon mainly to the right of the center axis is still observable. Whereas the peak values of up- and downdraft for all other configurations discussed before showed values of almost the same size for both levels at $z / H=1.0$ and 1.0833 , in the case of
$15 \mathrm{~m}$ tree crowns, a considerable difference is found. The velocity of the downdraft (downwind along wall B) in the lee of the treetop is substantially greater than the velocity of the updraft (upwind along wall A) in front of the tree top.

Comparing Figs. 6, 12 and 17 reveals with increasing crown volume a decreasing lateral horizontal inflow into the canyon near wall $\mathrm{B}$ and an increasing horizontal outflow near wall $\mathrm{A}$. That is, the net effect is a reduced inflow of fresh air, which explains the strong increase of concentration at the canyon ends with increasing crown diameter.

\subsubsection{Trees with spherical impermeable crowns of $15 \mathrm{~m}$ diameter and two-way traffic (TWT40)}

In a further measurement series, the street canyon with trees of $15 \mathrm{~m}$ crown diameter was subjected to two-way traffic by $40 \mathrm{~km} \mathrm{~h}^{-1}$. At Wall B, a significant reduction in concentration in the middle part (Fig. 18) can be seen again. Apparently, the traffic-induced turbulence is also compensating the blockade effect of the tree crowns to a certain extent at wall $\mathrm{A}$. This is due to the fact that the pollutants in the street volume beneath the tree crowns are mixed efficiently by the car movement, which leads to a more homogeneous concentration. This effect can be inferred also from the case of the $9 \mathrm{~m}$ tree crowns in Fig. 13. The equalizing effect of car movements and the associated traffic-induced turbulence can be seen also in Fig. 19, where the relative change between standing traffic and twoway traffic with $40 \mathrm{~km} \mathrm{~h}^{-1}$ is given for the canyon with trees of $15 \mathrm{~m}$ diameter crowns. 

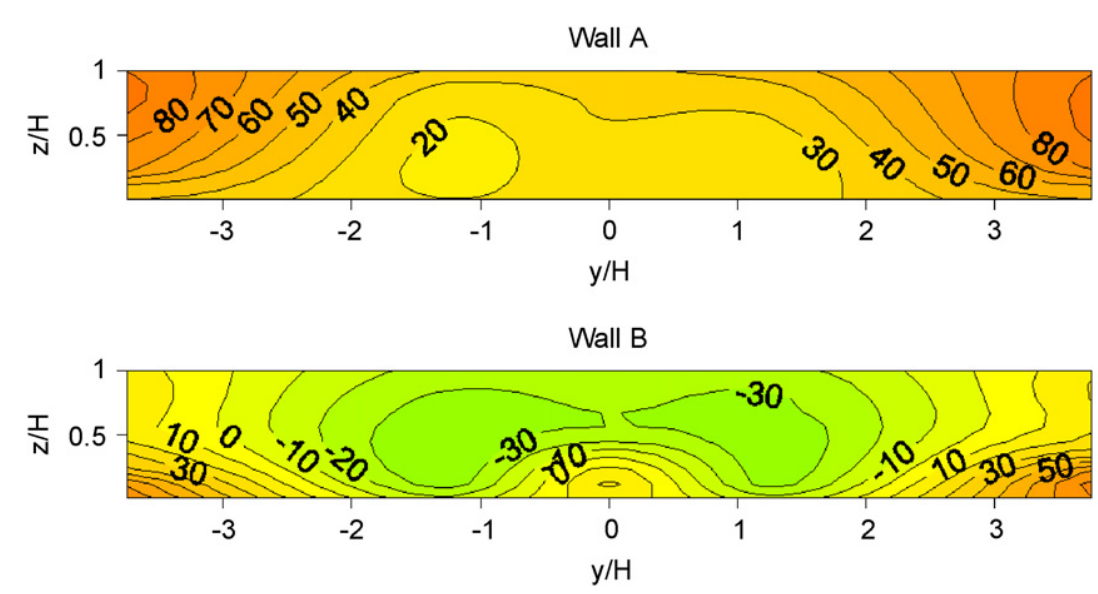

Fig. 15. Relative change in wall concentrations for street canyon with trees of $15 \mathrm{~m}$ crown diameter and standing traffic (ST00) when compared to no tree situation (Fig. 3).

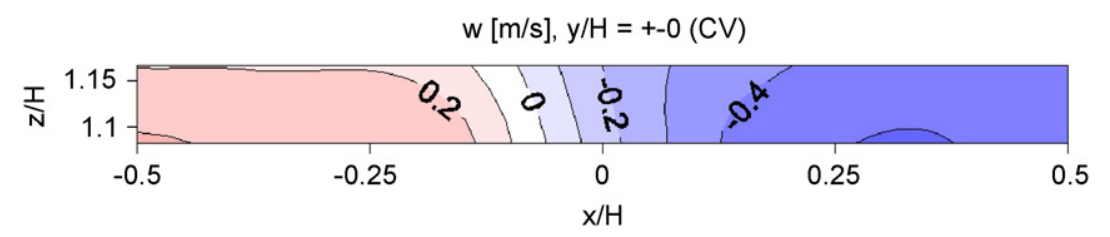

Fig. 16. Mean vertical velocity $w$ above street canyon with trees of crown diameter $15 \mathrm{~m}$ and standing traffic (ST00).

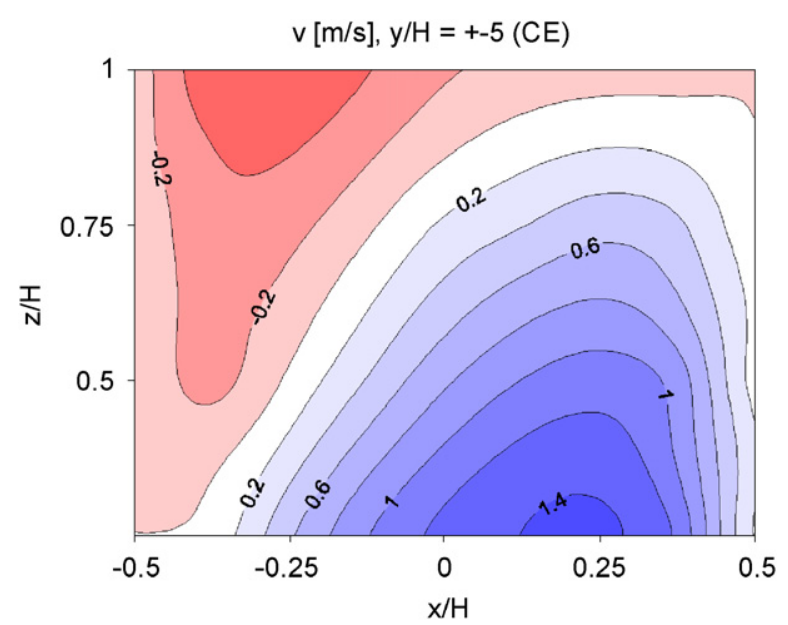

Fig. 17. Mean horizontal velocity $v$ at $y / H= \pm 5$ for street canyon with trees of crown diameter $15 \mathrm{~m}$ and standing traffic (ST00).

Concerning the flow field situation above and in the outermost cross section of the street canyon, it can be stated that almost no changes in comparison to the configuration of the previous section are detectible.

\subsection{Influence of tree crown permeability on concentration levels}

So far, all model configurations investigated were performed with impermeable spherical Styrofoam tree crowns. However, real tree crowns consist of stems, leaves and stalks and form permeable and flexible structures. Since critical concentration in street canyons are associated with low wind situations, the flexibility of the structure does not play an important role. In order to investigate the additional influence of crown permeability, spherical tree crowns with diameter $15 \mathrm{~m}$ have been manufactured from a homogeneous porous media (foam 10 ppi), see Fig. 20. The label 10 ppi stands for " 10 pores per inch" and is the most porous foam material available on the market. Measurements of porosity result in a relative pore volume of $97 \%$. A quantitative description of the material's permeability can be given by the loss in static pressure per unit length. Measurements of the pressure loss coefficient $\lambda$, i.e. of the static pressure loss $\Delta p_{\text {stat }}$ per material thickness $d$ normalized by the dynamic pressure $\Delta p_{\text {dyn }}$, yield values of $\lambda=210$ $275 \mathrm{~Pa}(\mathrm{~Pa} \mathrm{~m})^{-1}$. 

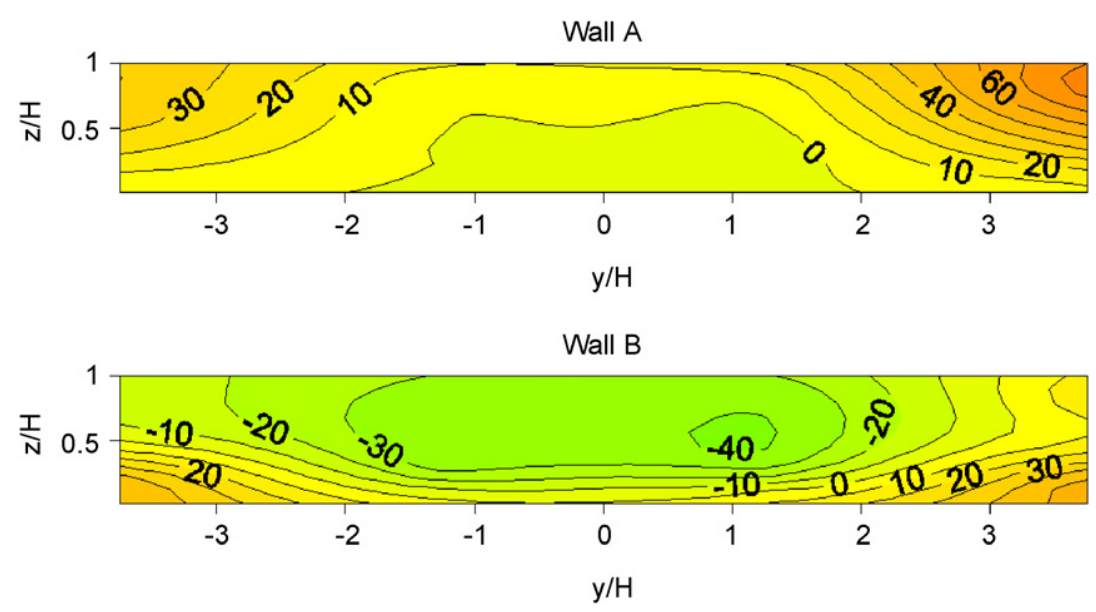

Fig. 18. Relative change in wall concentrations for street canyons with trees of crown diameter $15 \mathrm{~m}$ and two-way traffic (TWT40) when compared to no tree situation (Fig. 7).

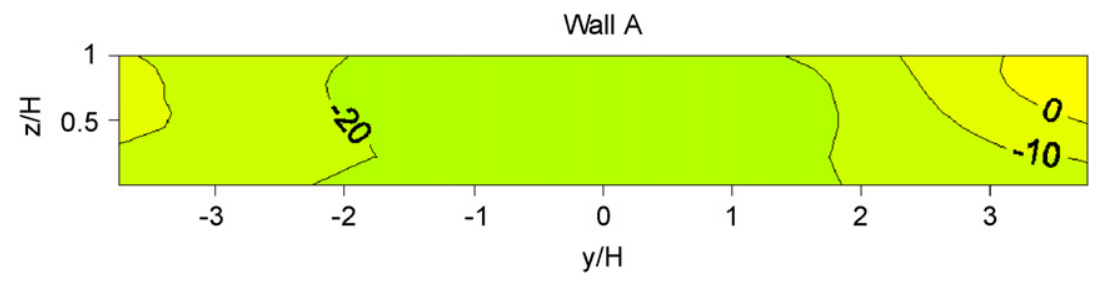

Fig. 19. Relative change in concentrations at wall A for trees with $15 \mathrm{~m}$ crown diameter and two-way traffic (TWT40) when compared to standing traffic (ST00) situation.

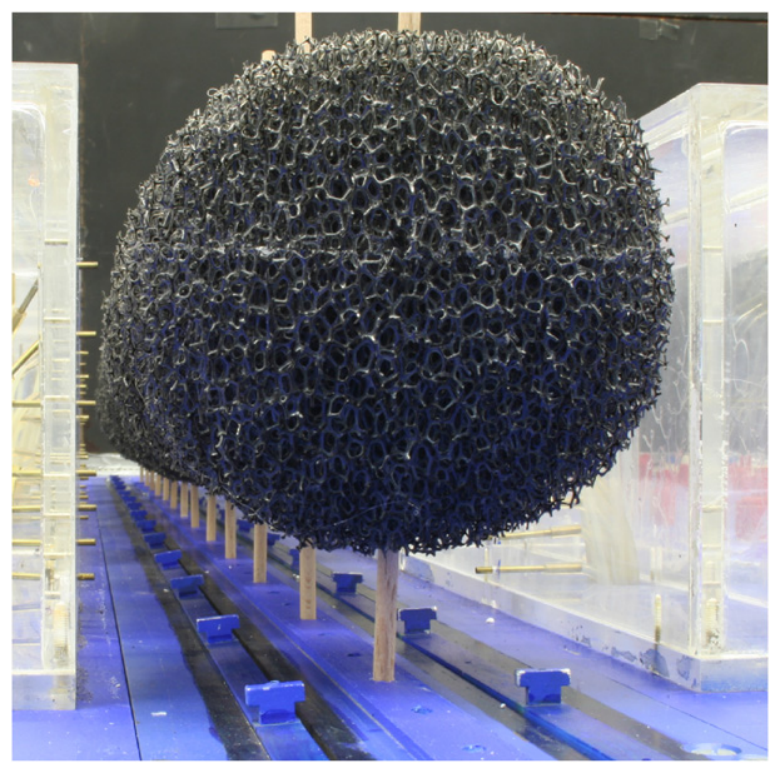

Fig. 20. Photograph of a tree crown made out of foam 10 ppi.

In Fig. 21, the percentage change of the dimensionless concentrations at wall A for permeable spherical crowns of diameter $15 \mathrm{~m}$ is shown when compared to impermeable Styrofoam crowns. The upper contour plot shows the results for the standing traffic, the lower plot the results for the two-way traffic case with $40 \mathrm{~km} \mathrm{~h}^{-1}$. No pronounced deviations of the concentration distributions are detectible in the plots and the overall averaged deviation amounts to only $+4.2 \%$ and $-7.8 \%$ for standing traffic and two-way traffic, respectively. This change is almost in the range of measurement accuracy, so that a substantial difference in the flow fields between impermeable and permeable tree crowns cannot be deduced (further variation of permeability will be performed currently).

\subsection{Influence of the height of the branch free trunk}

One of the parameters characterizing the tree planting configuration in a street canyon is the height of the branch free trunk extending from the floor to the lower end of the crown, in the following denoted as trunk height. Variations of the trunk height have been performed for impermeable crowns with diameter 9 and $12 \mathrm{~m}$ for standing 

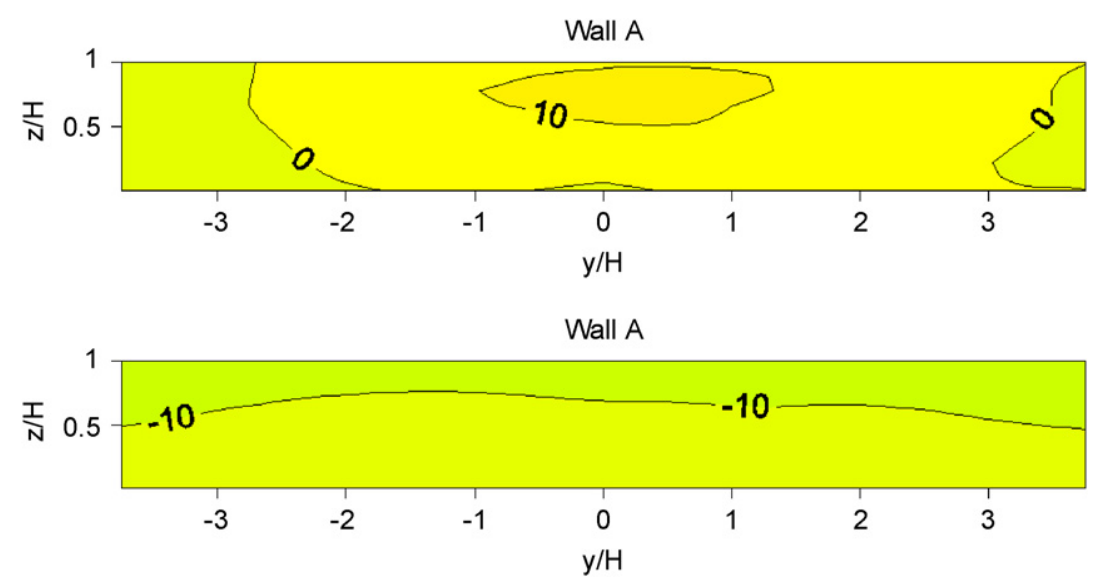

Fig. 21. Relative change in concentrations at wall A for permeable crowns when compared to impermeable crowns for standing traffic (ST00) (top) and two-way traffic (TWT40) (bottom).
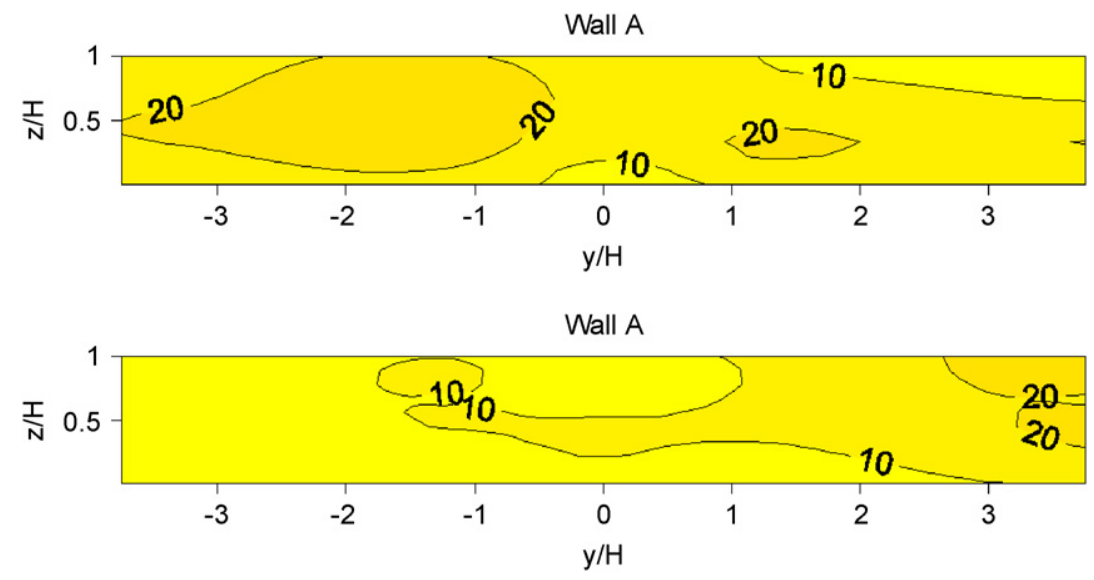

Fig. 22. Relative change in concentrations at wall A for trees with trunk height $9 \mathrm{~m}$ when compared to trunk height of $4.5 \mathrm{~m}$; crown diameter $12 \mathrm{~m}$, standing traffic (ST00) (top) and two-way traffic (TWT40) (bottom).

traffic as well as for two-way traffic motion with $40 \mathrm{~km} \mathrm{~h}^{-1}$.

In case of small sized tree crowns with $9 \mathrm{~m}$ diameter and a variation of trunk height between 4.5 and $9 \mathrm{~m}$, only negligible changes in concentration levels are detectible. If one averages the change in the whole wall $\mathrm{A}$, an average value of change of $0.9 \%$ for the standing traffic case and a value of $3.4 \%$ for the two-way traffic case with $40 \mathrm{~km} \mathrm{~h}^{-1}$ is computed.

In case of medium sized tree crowns with $12 \mathrm{~m}$ diameter (Fig. 22) a more pronounced change in concentration level at wall $\mathrm{A}$ is detectible with trunk height variation. The relative change in wall A concentration is displayed for standing traffic (top) and two-way traffic (bottom). In the figures, the change with $9 \mathrm{~m}$ trunk height is indicated when compared to the results with $4.5 \mathrm{~m}$ trunk height. The averaged concentration change for wall $\mathrm{A}$ amounts to $17.4 \%$ and $9.6 \%$ for standing and two-way traffic, respectively. With $9 \mathrm{~m}$ trunk height, the tree tops are exceeding the roof top level by $3 \mathrm{~m}$, while in the setup with only $4.5 \mathrm{~m}$ trunk height, the tree tops were $1.5 \mathrm{~m}$ below roof top level. This differentiates this configuration from the former one yielding somewhat different flow conditions in the canyon.

Keeping all other boundary conditions constant, the variation of the trunk height leads to a noticeable change in concentration at wall A, only if the tree tops overtop the canyon roof level. The latter changes the amount of entrained air into the canyon significantly. 

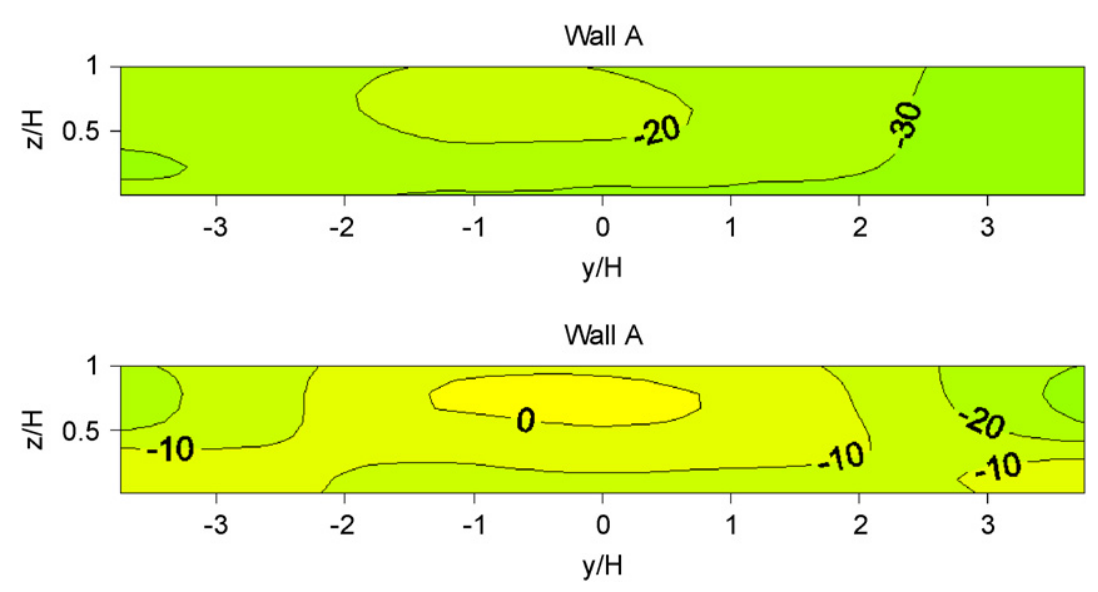

Fig. 23. Relative change in concentration at wall A for $20 \mathrm{~m}$ tree spacing when compared to $15 \mathrm{~m}$ tree spacing; crown diameter $15 \mathrm{~m}$, standing traffic (ST00) (top) and two-way traffic (TWT40) (bottom).

\subsection{Influence of tree spacing}

Finally, a further parameter of influence was studied-the spacing of tree planting. Measurements were carried out with impermeable, spherical Styrofoam tree crowns of diameter $15 \mathrm{~m}$ and the spacing was increased from 15 to $20 \mathrm{~m}$. The change in concentration in comparison to the corresponding setup with $15 \mathrm{~m}$ tree spacing measured at wall A for both, the standing and two-way traffic case with $40 \mathrm{~km} \mathrm{~h}^{-1}$, are depicted in Fig. 23.

With this configuration, the tree crowns occupied $27.3 \%$ of the street canyon volume. Wall A clearly shows a reduction in concentration. The wall averaged change of tracer gas concentration amounts to $-25.8 \%$ for standing traffic and to $-9.4 \%$ for the two-way traffic case. Towards the ends of the street canyon, an even stronger decrease in concentration is detected. Apparently, a larger tree spacing decreases the concentrations, as expected. Thus, tree spacing is an important factor to be considered for natural ventilation of street canyons.

\section{Discussion}

For the investigations concerning the influence of crown diameter on flow and pollutant dispersion phenomena (see Section 3.2), an overview of wall averaged dimensionless tracer gas concentrations is given in Fig. 24. Additionally, concentration values for model investigations with medium sized crowns of $12 \mathrm{~m}$ diameter, which have not been presented here, are included.

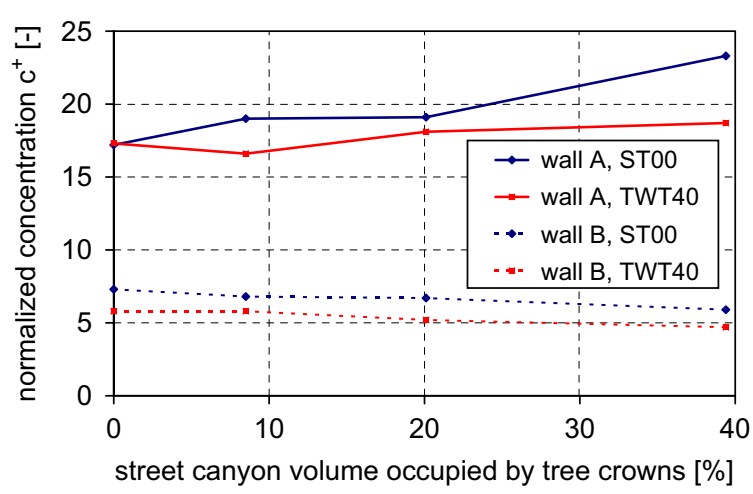

Fig. 24. Wall averaged dimensionless concentration values.

Regarding wall A, a steady increase in concentration is registered with increasing crown volume. In the standing traffic case, an enhanced increase in tracer gas concentration is clearly visible in comparison to the moving traffic case. With traffic motion, pollutants are not only distributed more homogeneously but also carried out of the canyon more effectively. Note that for the no-tree setup no difference for the averaged concentration value of wall $\mathrm{A}$ is noticeable. In contrast, wall $\mathrm{B}$ shows a small decrease in pollution concentration for increasing crown diameters. Again, somewhat lower concentration values can be seen in the case of moving traffic. All in all, tree planting increases the integral concentrations at the leeward wall $\mathrm{A}$ and lowers slightly the integral concentration at the windward wall $\mathrm{B}$. 


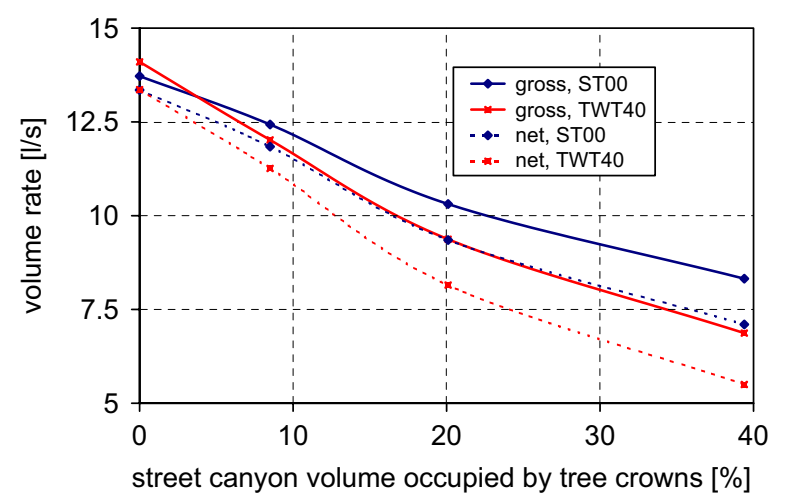

Fig. 25. Volume rates of flow entering the street canyon horizontally through cross section at $y / H=-5$.

In Fig. 25, the gross and net volume rates of flow entering the street canyon horizontally in street axis direction through the outermost cross section at $y / H=-5$ are shown. The gross flow rate (solid lines) denotes the total amount of flow entering the canyon, whereas in the net flow rate (dashed lines) the flow leaving the canyon through the cross section is subtracted from the gross flow rate. As expected, a continuous decrease of gross and net flow rate is registered with an increased blockade effect of larger tree crowns.

Summarizing the influence of the crown diameter, it can be stated that in the case of small tree crowns significant relative increases in concentration are measured only at the street ends, since the corner eddies are hindered in entering the street canyon. However, no pronounced changes are observable in the middle part of the canyon, indicating the existence of a canyon vortex or a canyon vortexlike structure. Increasing crown diameters yield to enhanced concentration levels with considerable increase even in the middle part of the canyon.

Furthermore, the volume rate of flow entering horizontally the canyon by passing the outermost cross sections is significantly reduced by blockage effects of large crowns. Substituting impermeable tree crowns by permeable crowns, showed for the permeability chosen (porous foam $10 \mathrm{ppi}$ ) no significant changes in the flow field and in wall concentrations. However, further grades of permeability have to be investigated in order to clarify the influence of crown permeability definitely.

The influence of tree trunk height on the concentration field is of secondary order except for the case that the tree tops exceed the roof level of the canyon. When the tree trunk height is changed, such that tree tops overlap the roof level by remaining all other parameters constant, higher pollutant concentrations occur at wall A.

Varying the tree spacing seems to be a very effective measure to influence the concentration level inside the street canyon. In the standing traffic case, an increase of tree spacing by $33 \%$ was accompanied by a change of the wall-averaged concentration by $-25.8 \%$ at wall $\mathrm{A}$. Moreover, the reduction of pollutant concentration at wall $\mathrm{A}$ is distributed more homogenously.

In regard to the situation at the pedestrian level in the canyon middle part, it can be stated, that the concentration levels in the lower part are not strongly affected by tree plantings. In contrary, most concentration plots show fewer changes in the lower wall part in comparison to the upper part.

Traffic-induced turbulence of two-way traffic motion reduces the averaged concentration level and causes a more homogeneous distribution of pollutants in the canyon, i.e., moving traffic improves the mixing of pollutants. The presence of large tree crowns subdivide the lower from the upper half of the canyon and a channeling effect of traffic-induced air motion is evident. Here, an interaction between traffic-induced turbulence and crown diameter is observable. Regarding Figs. 24 and 25 reveals a broader gap between the standing and moving traffic case for larger tree crowns. This means, that the impact of the traffic on the flow and concentration field is enhanced in presence of larger tree crowns.

A comparison of this study with numerical results from Ries and Eichhorn (2001) is restricted to some general flow and concentration characteristics. Ries and Eichhorn (2001) calculated the flow and concentration field inside a street canyon with an aspect ratio $H / W=0.47$, without including trafficinduced turbulence. Inside the canyon two rows of trees with crown diameter of $4 \mathrm{~m}$ and separated by a $14 \mathrm{~m}$ broad traffic lane were arranged. Since they applied a two-dimensional model, their results do not account for effects induced by sidewise entering corner eddies, but only for the middle part of a sufficient large street canyon (i.e. $L / H>7$ ), where the flow field shows no three-dimensional behavior. Despite all these differences to the model configurations investigated in this study, some common flow and concentration field characteristics are present. With regard to the concentration field, 
they found increased values at wall $\mathrm{A}$ and decreased values at wall $\mathrm{B}$ when compared to the street canyon without trees. Moreover, their results show a slight increase of flow velocities in front of wall B and decrease in front of wall $\mathrm{A}$.

\section{Conclusion}

Maximum dimensionless concentrations were always measured in the middle part of the canyon at wall $\mathrm{A}$. This holds for all configurations investigated with and without trees as well as standing traffic and two-way traffic. A single row of trees planted at the street center axis leads to higher wall-averaged concentrations at wall $\mathrm{A}$ and to somewhat lower values at wall $\mathrm{B}$. The influence of tree planting is particularly noticeable at both walls $\mathrm{A}$ and $\mathrm{B}$ towards the street ends where the highest rise in concentration occurs.

The results presented in this paper allow to formulate first recommendations for city planners in order to account for the health and quality of life of urbanities. Tree crowns should not occupy large canyon volumes in order not to suppress the ventilating canyon vortex system and the corner eddies. In particular, sufficient free space between crowns and adjacent walls should be ensured. Otherwise, the air exchange is hindered and the concentration of pollutants rises in the pedestrian level. Further attention has to be paid to the total tree height, which should not exceed the roof top level leading to a substantial reduction of entrained air. A broader tree spacing ensures a better ventilation of the canyon, since the vortices generated by the atmospheric overflow can better intrude into the canyon and remove the polluted air.

\section{Acknowledgement}

The financial support of the Deutsche Forschungsgemeinschaft DFG for the presented study (grant no. Ru 345/28-1) is gratefully acknowledged by the authors.

\section{References}

Ahmad, K., Khare, M., Chaudhry, K.K., 2005. Wind tunnel simulation studies on dispersion at urban street canyons and intersections - a review. Journal of Wind Engineering and Industrial Aerodynamics 93, 697-717.

Baik, J.J., Kim, J.J., 1999. A numerical study of flow and pollutant dispersion characteristics in urban street canyons. Journal of Applied Meteorology 38, 1576-1589.

Chang, H.-C., Meroney, R.N., 2003. Concentration and flow distribution in urban street canyons: wind tunnel and computational data. Journal of Wind Engineering and Industrial Aerodynamics 91, 1141-1154.

Eliasson, I., Offerle, B., Grimmond, C.S.B., Lindqvist, S., 2006. Wind fields and turbulence statistics in an urban street canyon. Atmospheric Environment 40, 1-16.

Gayev, Y.A., Savory, E., 1999. Influence of street obstructions on flow processes within urban canyons. Journal of Wind Engineering and Industrial Aerodynamics 82, 89-103.

Gerdes, F., Olivari, D., 1999. Analysis of pollutant dispersion in an urban street canyon. Journal of Wind Engineering and Industrial Aerodynamics 82, 105-124.

Gromke, C., Ruck, B., 2005. Die Simulation atmosphärischer Grenzschichten in Windkanälen. In: Proceedings 13th GALA Fachtagung Lasermethoden in der Strömungsmechanik, German Association for Laser Anemometry, pp. 51-1-51-8.

Hunter, L.J., Watson, I.D., Johnson, G.T., 1990/91. Modelling air flow regimes in urban canyons. Energy and Building 15-16, 315-324.

Kastner-Klein, P., Plate, E., 1999. Wind-tunnel study of concentration fields in street canyons. Atmospheric Environment 33, 3973-3979.

Kastner-Klein, P., Fedorovich, E., Rotach, M.W., 2001. A wind tunnel study of organised and turbulent air motions in urban street canyons. Journal of Wind Engineering and Industrial Aerodynamics 89, 849-861.

Meroney, R., Pavageau, M., Rafailidis, S., Schatzmann, M., 1996. Study of line source characteristics for 2-D physical modelling of pollutant dispersion in street canyons. Journal of Wind Engineering and Industrial Aerodynamics 62, 279-290.

Oke, T.R., 1988. Street design and urban canopy climate. Energy and Buildings 11, 103-113.

Plate, E., 1982. Windkanalmodellierung von Ausbreitungsvorgängen in Stadtgebieten. In: Abgasbelastungen durch den Kraftfahrzeugverkehr, Verlag TÜV Rheinland $\mathrm{GmbH}$, pp. 61-83.

Ries, K., Eichhorn, J., 2001. Simulation of effects of vegetation on the dispersion of pollutants in street canyons. Meteorologische Zeitschrift 10 (4), 229-233.

So, E.S.P., Chan, A.T.Y., Wong, A.Y.T., 2005. Large-eddy simulations of wind and pollutant dispersion in a street canyon. Atmospheric Environment 39, 3573-3582.

Vardoulakis, S., Fisher, B.E.A., Pericleous, K., Gonzalez-Flesca, N., 2003. Modelling air quality in street canyons: a review. Atmospheric Environment 37, 155-182. 